\title{
Continuity and change in preferred provider organizations
}

\section{Thomas Rice', Jon Gabel'2, Stephen Mick', Clare Lippert ${ }^{2}$ and Colleen Dowd ${ }^{4}$}

\author{
'University of North Carolina School of Public Health, Chapel Hill, NC, \\ ${ }^{2}$ Health Insurance Association of America, Washington, DC, ${ }^{3}$ University of \\ Michigan School of Public Health, Ann Arbor, MI, and ${ }^{4}$ Donelson Hospital, \\ Nashville, TN, U.S.A.
}

\section{Summary}

This paper presents the results from a national survey of preferred provider organizations (PPOs) that was conducted in 1988. It is based on telephone interviews conducted by the authors with executives in over 170 PPOs in the United States. We compare the survey results with those obtained from similar surveys conducted In 1985 and 1986, allowing us to assess the extent to which PPOs have grown and changed. We found that PPOs have continued to grow at an extremely rapid rate. During the Summer and Fall of 1988, the time in which the survey took place, 37.6 million people were ellgible to use PPO benefits, compared to the 16.5 million figure we obtained two years earlier. We did not find, however, that PPOs are moving in the direction of providing more innovative forms of health care cost containment. Most PPOs still rely on discounts from providers and utlization review to achieve savings. There is little trend towards using incentive reimbursement techniques and choosing preferred providers that have shown themselves to be cost-efficient. We conclude that in the coming years PPOs must demonstrate the ability to control rising health care costs. To accomplish this, they will need to put more pressure on providers to use resources more sparingly. Otherwise, they may lose their market share to other forms of managed care.

PPOs; Cost-containment; Incentive relmbursement; U.S.A.

Address for correspondence: Professor Thomas Rice, Department of Health Policy and Administration, School of Public Health, University of North Carolina, 263 Rosenau Bldg, CB\# 7400, Chapel Hill, NC 27514, U.S.A. 


\section{Introduction}

This paper presents the results from a national survey of Preferred Provider Organization (PPO) that was conducted in 1988. The study, sponsored by the Health Insurance Association of America (HIAA), was based on telephone interviews with executives in over 170 PPOs. We compared the survey results with those obtained from similar surveys conducted in 1985 and 1986, allowing us to assess the extent to which PPOs have grown and changed.

PPOs are arrangements in which a defined group of health care providers agrees to provide services to a defined group of individuals. Patients are not 'locked into' using the preferred providers, and therein lies one of the differences between PPOs and Health Maintenance Organizations [1]. Usually patients are given financial incentives to use the provider panel. In return for obtaining 'preferred' status (which is designed to increase the volume of business), providers make their services more attractive to payers through means such as discounting, utilization review, and perhaps, practicing medicine in a way that conserves medical resources. PPOs are sponsored by a variety of organizations, including providers, insurers, and other health care firms.

PPOs have grown at an astounding rate. Previous studies conducted by the authors indicate that the number of individuals eligible to use PPOs has risen from 1.3 in December 1984 to 5.8 million in the summer of 1985 , to 16.5 million in the summer of 1986 [2]. The results from the current survey indicate that these numbers may have doubled in the ensuing two years.

The reasons for PPO growth are not difficult to find. Employers, who are the major purchasers of health care benefits for the working age population, have become increasingly frustrated with the rising health insurance premiums under their conventional health care plans. HMOs, once thought to be a panacea, have not solved the problem. Even if HMOs do conserve medical care resources, employers typically have not shared in these savings because, until recently, they were usually required to make equal contributions to all plans. Furthermore, although there is conflicting evidence concerning whether HMO enrollees are healthier than those who choose to remain in the fee-for-service sector, there is still some evidence to indicate that lower-risk individuals are attracted to HMOs. According to Hellinger, this may be because those who are attracted by HMOs' emphasis on preventive services are 'parsimonious users of medical services' [3].

Many patients do not like the lock-in features of HMOs. By offering patients a choice of providers, PPOs place fewer roadblocks in the way of patients when they enter the medical care system. Recently, the Health Care Financing Administration began a large demonstration project to test whether the concept can be incorporated into the Medicare program's structure, one more sign of the growing interest in the PPO concept [4].

There is a 'down-side' to PPOs, of course: doubt as to whether they are able to successfully control health care inflation rates. There is reason to be skeptical about whether PPOs can achieve savings. By offering patients lower out-of-pocket prices than would be paid under traditional fee-for-service plans, PPOs might actually 
increase service utilization. Thus, in order to save money, they must employ strong counteracting measures, such as regulatory protocols or provider incentives designed to control utilization.

Previous researchers have not been able to determine the extent (if any) to which PPOs reduce medical care costs. There have been only two comprehensive studies that have employed detailed claims data, one conducted by Hester, Wouters and Wright [5], and the other by Hosek, Marquis, Garnick and Luft [6]. The number of employer groups (and therefore, PPOs) studied, however, was very limited: one in the former and five in the latter.

The Hester study examined the impact of one California PPO at one large firm. Using detailed health claims data, the authors found that although employee expenditures were lower for those using the PPO, this resulted largely because users tended to be much healthier than other employees and their dependents. Furthermore, users tended to frequent the preferred providers mostly for minor illnesses.

The Hosek study examined the experience of five employers with PPOs, all of which were operational by 1983. The study compared the utilization of individuals who used the PPO as their regular source of care with those that did not. The authors found that PPO participants did not differ very much from non-participants in terms of health status or prior use of services. For most of the employers, total medical care payments for PPO participants were less than for non-participants, largely due to lower numbers of visits outside of the hospital. However, when examining episodes of illness, it was found that PPO patients had higher charges, largely because they had more physician consultations, probably due to lower coinsurance rates [7]. Combined, the two studies do not provide strong evidence about the ability of PPOs to control costs.

Our previous studies of PPOs led us to make a number of predictions about how they would evolve. In general, we concluded that their success would hinge on their ability to control costs. In particular, we concluded that provider-sponsored PPOs would have the most difficulty succeeding, because it would be difficult for the PPO to control utilization when its member hospitals and/or physicians had a financial incentive to fill beds or increase the number or complexity of services provided [8]. Conversely, PPOs sponsored by insurers (commercials and the Blue Cross/Blue Shield plans) and investor firms would be likely to have more of a stake in controlling utilization.

Second, we suspected that successful PPOs would be those that provided some extra incentive to control utilization, either through selecting cost-conscious providers, or using payment mechanisms that encourage the conservation of resources (e.g., DRGs for hospitals, 'managed care' for outpatient services) [9-11]. Third, we predicted that successful PPOs would be ones that developed strong data bases for both choosing and monitoring the utilization activity of providers [12]. This would include not only detailed hospitalization data, but also claims information or profiles on physicians as well.

Finally, we anticipated that there would continue to be large growth in PPO arrangements [13]. Much of the reason was that as of 1986, PPOs had not penetrated 
many parts of the country, and, furthermore, many commercial insurers and Blue Cross/Blue Shield plans had just begun to organize PPOs. Once fully in operation, these organizations could bring very large numbers of people into the plans. The extent to which PPOs have or have not met these expectations is discussed below.

\section{Survey methodology}

In conducting the survey, we employed a methodology similar to that used in the PPO surveys we carried out in 1985 and 1986 . We personally conducted telephone interviews with executives in each of 178 PPOs during the Summer and Fall of 1988. The interviews ranged from 10 minutes to an hour in length, and typically lasted about 20 minutes. The response rate was $100 \%$ for insurer-sponsored plans, and $86 \%$ for the others.

To obtain a sampling frame for the study, we included in our universe all PPOs that were operational as of 31 December 1987, except for those that provided only a single type of medical service (such as prescription drugs or foot care). We obtained a listing of all PPOs in the United States from three sources: The June 1988 Directory of the American Medical Care and Review Organization [14]; a listing of Blue Cross and Blue Shield plans that had PPOs; and internal records of HIAA.

We drew the sample in three stages. First, based on the HIAA records, all member companies with PPOs were included in the sample (a total of 60). All other sponsorship categories underwent the next two stages. PPOs with more than 100000 eligibles, based on information from AMCRA and our 1986 survey, were automatically included in the sample. Including all of the largest PPOs allows us to account for the majority of the population that is eligible to use PPOs. The final step was to draw a random sample of PPOs with expected eligibility figures of less than 100000 .

The number of PPOs to interview within each of the sponsorship groups was based on the proportion of all PPOs (not eligibles) thought to be in each of these groups [15]. Although published figures and sponsorship classifications (as well as past survey information) were used to select the sample, weights for the random portion of the sample were based on the (more accurate) data obtained in the interviews. Results depicting the number of eligibles (Figs. 1 and 2) are weighted to represent the PPO universe, whereas the other figures and tables are unweighted to allow for comparison of the results to those obtained from our 1986 (unweighted) data.

Although we surveyed 178 PPOs, the results that follow are based on a sample of 147 [16]. Thirty-one interviews were eliminated because they may have been duplicative. These PPOs were sponsored by commercial insurance companies that did not operate their own PPO, but instead contracted with established PPOs in order to use their provider networks. Since our sample also included the latter organizations, we chose not to use results from the 31 interviews, to avoid doublecounting. 


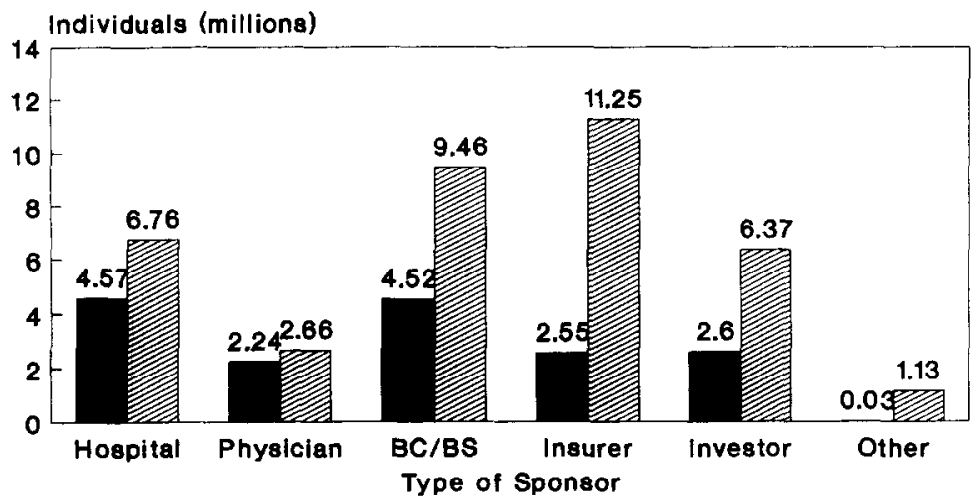

Fig. 1 Number of Individuals eligible to use PPOs in 1986 and 1988, by sponsor. Solid bars, 1986; hatched bars, 1988.

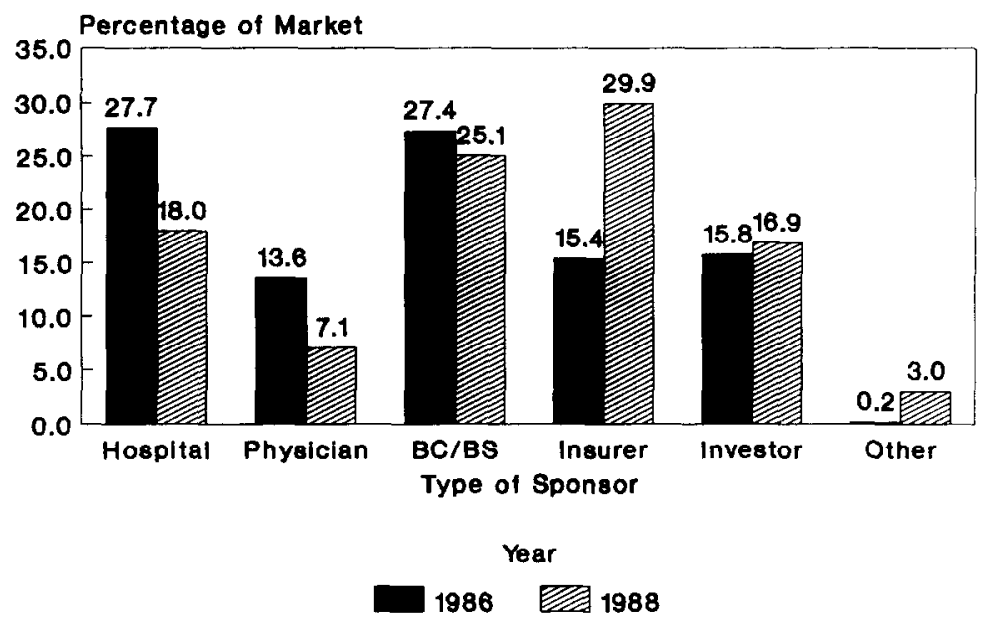

Fig. 2 Share of PPO market in 1986 and 1988, by sponsor. Solid bars, 1986; hatched bars 1988.

\section{Findings}

Number of individuals eligible to use PPOs PPOs have continued to grow at an extremely rapid rate. During the summer of 1988 , the time in which the survey took place, respondents reported that the total number of employees and dependents eligible to use PPO benefits was 37.6 million, compared to the 16.5 million figure we obtained two years earlier. This figure is quite close to the 33.9 million figure obtained by AMCRA in a questionnaire mailed in June 1988 [17].

Eligibility figures were calculated for each sponsorship category according to a formula that took into account the two stage sampling process [18]. The total eligibility figure must be interpreted cautiously for a number of reasons. First, it 
is possible that PPOs overestimate the number of eligibles, although it should be pointed out that there is little reason to expect that they would err more than in previous years.

Second, there is some possibility of double-counting if PPOs are in a joint venture with each other, and we report total eligibility as the sum of the two. We have attempted to correct for this problem by eliminating insurers that purchased PPO networks, as discussed above. Of the remaining 147 PPOs we surveyed, about one-fourth reported some joint ventures, and most of those were not with other PPOs. Consequently, the amount of upward bias in the figures that results from double-counting should be small.

Finally, the 37.6 million figure shows the number of individuals who are eligible to use PPO benefits, not the number who actually do so. In some employer health insurance plans, individuals and their families are automatically enrolled in the PPO. However, they may continue to see their own providers even if the providers are not in the network, although in doing so the patients usually forgo lower copayments. As an indication of the extent to which this occurs, the study reported on earlier by Hester, Wouters and Wright found that $62 \%$ of eligibles did not use the study PPO at all during 1984 [19].

In fact, the figures we obtained are considerably higher than those that are obtained when one surveys employers rather than PPOs. In a national survey of employee benefit managers that we conducted during the Spring of 1988, it was found that $11 \%$ of U.S. employees and dependents were in PP0s [20]; this implies a total enrollment of less than 20 million people. We have already given several reasons why our 37.6 million figure may be an overestimate. One minor reason as to why the 20 million figure may be low is that it was based on a survey conducted several months before the PPO survey. During the year between our 1988 and 1989 employee surveys, there was substantial growth (in the order of $20 \%$ annually) in the number of firms offering PPOs. Nevertheless, this would account for only a fraction of the difference between the two numbers. Although it is likely that figures such as ours and AMCRA's are indeed overestimates, so long as PPOs overestimated their figures in the past as well as currently, the rates of growth would be unchanged.

With these caveats in mind, we can explore the eligibility figures in greater detail. Throughout the remainder of the paper, we divide PPOs into several mutually exclusive sponsorship categories: hospital, which includes PPOs sponsored by hospitals, those sponsored jointly by hospitals and physicians, as well as corporate hospital chains; physician, which encompasses those sponsored both by physicians and medical groups, such as Foundations for Medical Care (FMCs) in California; Blue Cross/Blue Shield; commercial insurer; investor, which is composed of entrepreneurial organizations other than hospitals, physicians, and commercial insurers, including third-party administrators (TPAs); and other, which includes 12 PPOs that do not fit into the previous categories: those sponsored by community groups, HMOs, and employers.

Fig. 1 shows the total number of individuals eligible to use PPOs during 1986 and 1988 by PPO sponsorship. All sponsorship types except physician-sponsored plans 
grew in absolute size during the previous two years. In fact, all of the non-provider categories more than doubled, which supports our expectation that PPOs with the most incentive to control health care cost inflation would be most likely to prosper.

The rate of growth of commercial insurer plans dominates those experienced by PPOs in the other sponsorship categories. Whereas in 1986 these PPOs were relatively unimportant, by 1988 they had the highest number of eligibles - over 11 million. (HIAA data show that the increase in the number of eligibles over these two years was mostly due to an increase in activity among those companies that already operated a PPO. In fact, a number of insurers are turning all of their group policy business into PPO-type plans. Between 1986 and 1988, the number of insurers with a PPO product rose only from 57 to 70 [21].) The emerging dominance of insurer and BC/BS plans in the PPO market is indicative of the changes we have reported in the group health insurance market [22]. Conventional insurance plans that offered little hope for cost control are quickly being replaced by plans that attempt to 'manage' the care provided to subscribers. PPOs offer a mechanism by which employers and their insurers or TPAs can wrestle some of the control over skyrocketing costs from the providers of care.

The changes in the composition of the PPO market are illustrated further in Fig. 2 , which shows how market shares have changed among sponsorship categories between 1986 and 1988. This figure highlights the extent to which providersponsored plans are being replaced as the dominant type of PPO. Whereas hospitalsponsored PPOs had the highest market share in 1986, two years later they were a distant third, barely having an edge of investor-sponsored plans. Similarly, physician-sponsored PPOs had plummeted from $17 \%$ to only $7 \%$ of the market.

One of the most impressive trends shown in Figs. 1 and 2 is the continued growth of investor-owned PPOs. Despite the dramatic growth in BC/BS and insurersponsored plans, investor PPOs garnered an even larger market share in 1988 than they did in 1986. As noted earlier, they are beginning to eclipse hospital-sponsored plans for third place. Although (or perhaps because) many of these PPOs did not start with an already established network of providers (unlike hospital and physician-sponsored plans) or subscribers (unlike the Blues and the insurers), they have developed tailor-made products for employers that are becoming increasingly popular.

Fig. 3 examines the number of individuals eligible to use the typical or median PPO, both in 1986 and 1988. The median PPO grew substantially in all ownership categories, which perhaps is not that surprising since many PPOs were just starting up during the earlier survey. We were surprised at the size of the PPOs we surveyed. Blue Cross/Blue Shield plans averaged almost 250000 members, and the commercial insurers had over 150000 . These figures should be viewed as having an upward bias, however, because we oversampled large PPOs. But since this was true also during our 1986 survey, growth rates should be unaffected.

\section{Scope of operations}

Table 1 shows the different geographic markets served by different types of PPOs, and how this changed between 1986 and 1988. Looking first at the more 


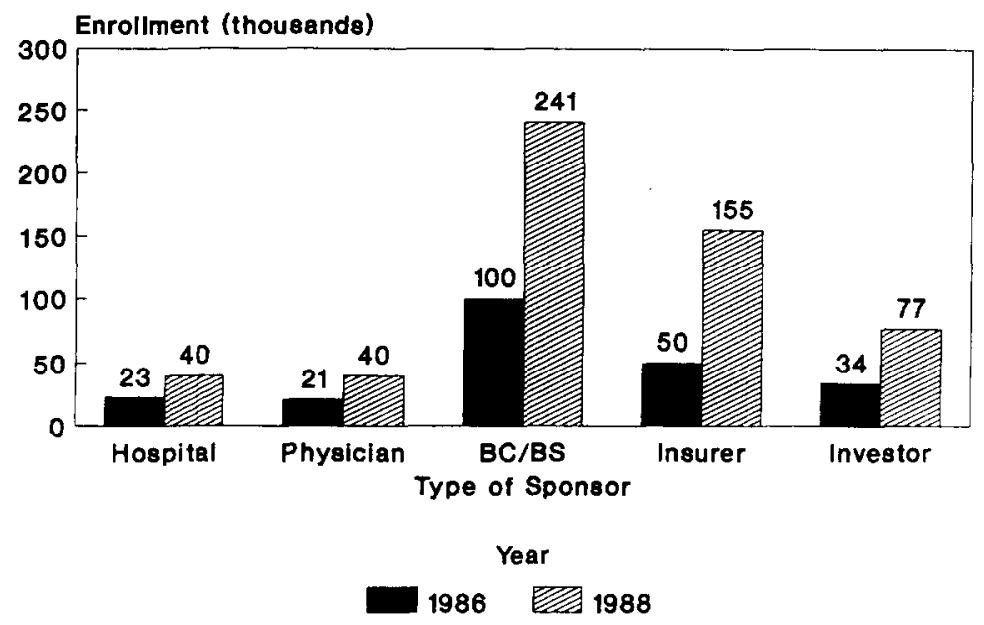

Fig. 3 Median number of Individuals eligible to use PPOs in 1986 and 1988, by sponsor. Solld bars, 1986; hatchod bars, 1988.

recent figures, it is not surprising that almost $80 \%$ of commercial insurer-sponsored PPOs serve two or more states, whereas most BC/BS plans serve a single state. On the other hand, both types of provider-sponsored plans tend to serve areas smaller than a state. Investor-sponsored plans fall between the provider and insurer sponsored plans.

What is noteworthy, however, is how all types of PPOs have expanded their geographic scope in only a two-year period. For example, whereas $75 \%$ of physiciansponsored PPOs served a single metropolitan area in 1986, this had dropped to $40 \%$ by 1988 . Similarly, while only $12 \%$ of hospital-sponsored PPOs served statewide or multi-state areas during 1986 , this almost tripled, to $32 \%$, two years later. Since this is a far faster rate of growth than is occurring in multi-hospital systems, it

Table 1

Geographic markets served by PPOs during 1986 and 1988, by sponsor (percentage)

\begin{tabular}{llrrr}
\hline Sponsor & $\begin{array}{l}\text { Single metro- } \\
\text { politan area }\end{array}$ & Sub-state & Statewide & Two or more states \\
\hline 1988 & & & & \\
Hospital & 31 & 37 & 14 & 18 \\
Physician & 40 & 30 & 20 & 10 \\
BC/BS & 5 & 26 & 63 & 5 \\
Insurer & 10 & 7 & 3 & 79 \\
Investor & 19 & 19 & 19 & 44 \\
l986 & & & & \\
& & & & 7 \\
Hospital & 51 & 37 & 5 & 0 \\
Physician & 75 & 19 & 6 & 0 \\
BC/BS & 4 & 26 & 70 & 25 \\
Insurer & 23 & 0 & 23 & 21 \\
Investor & 31 & 13 & 31 & \\
\hline
\end{tabular}


implies that consortia of hospitals are forming PPO networks. Insurers expanded to several states as well: $79 \%$ of the commercial insurer-sponsored plans served two or more states in 1988 , up from $54 \%$ two years earlier. These figures indicate that PPO organizations are becoming more national in scope, further implying that those PPOs that survive are likely to be those serving several geographic areas. In fact, larger PPOs may enjoy a variety of advantages in areas such provider selection, rate negotiation, and utilization review, where sophisticated data processing systems are particularly advantageous.

It follows that if PPOs expand their geographic boundaries, they are likely to spread more evenly throughout the country. In our first national PPO survey, conducted in 1985 , we found that $61 \%$ of those eligible to use PPOs lived in California, Colorado and Florida. Although our current survey did not collect parallel data, we were able to ascertain that PPOs have spread to many more parts of the country. When asked the primary state served, 33 states were listed by the 146 responding PPOs and 41 were listed as one of the three primary states served. (Had we surveyed all PPOs in the country, undoubtedly the figure would have been higher. AMCRA reports that 43 states and the District of Columbia contain or headquarter PPOs [23].) Although the three aforementioned states still garnered the largest number (53/146), several other states were listed by five or more PPOs: Illinois, Minnesota, Missouri, Ohio, Pennsylvania, Tennessee, Texas and Washington. We previously reported relatively little PPO activity in such population centers as New York, Pennsylvania and Texas. Although we still found relatively little activity in New York, the PPO movement appears to be growing in the other two states.

Another aspect of PPOs' scope of operations is shown by the sizes of their provider networks. Fig. 4 shows the median number of hospitals per PPO during 1986 and 1988, and Fig. 5 shows similar figures for the number of physicians. Given their greater sizes, it is not surprising that $\mathrm{BC} / \mathrm{BS}$ and commercial insurer-

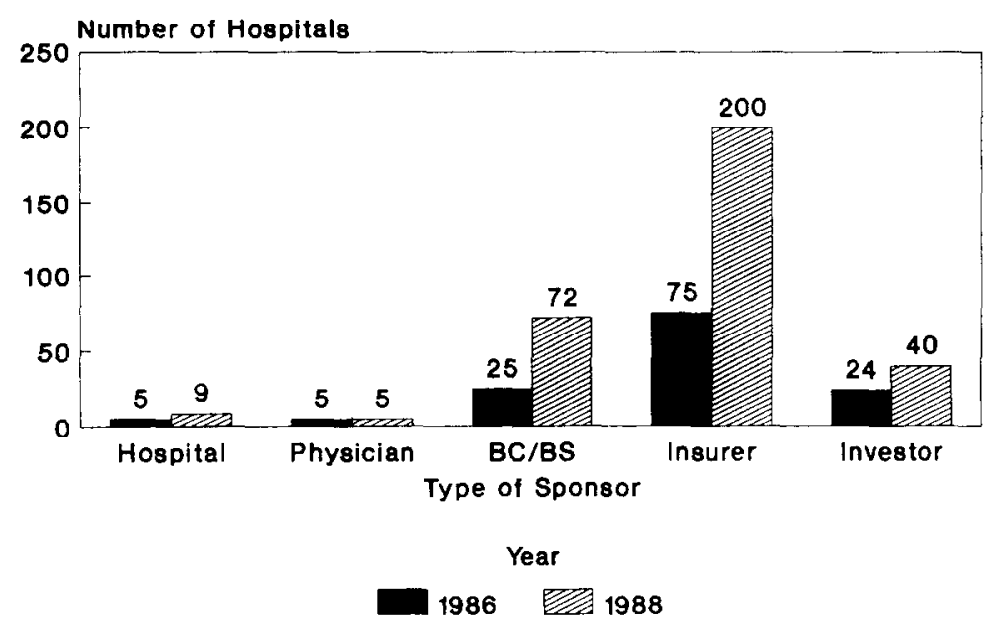

Fig. 4 Median number of hospltals per PPO in 1986 and 1988, by sponsor. Solid bars, 1986; hatched bars, 1988. 


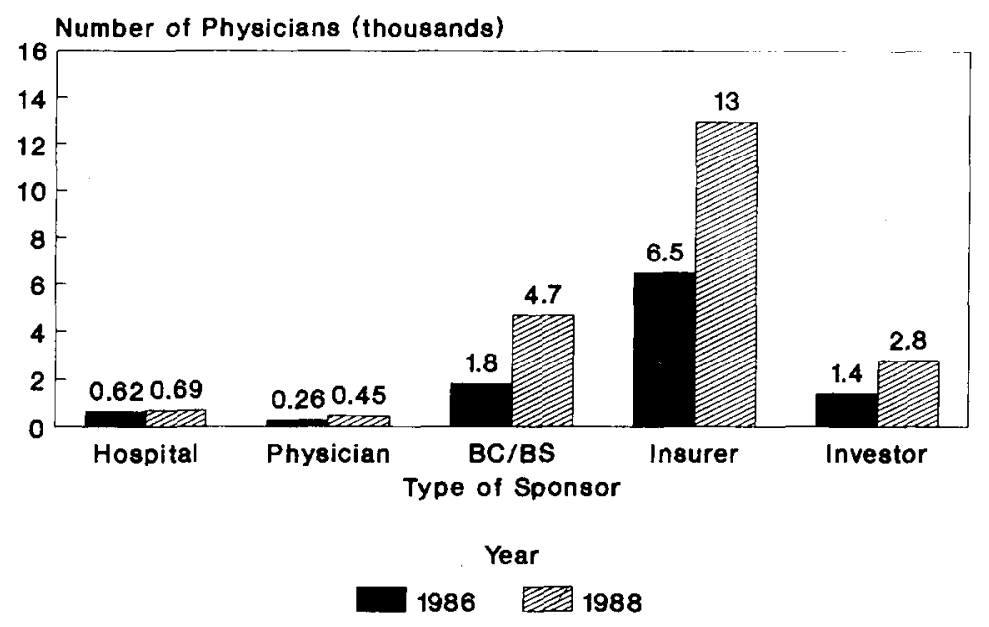

Fig. 5 Median number of physicians per PPO in 1986 and 1988, by sponsor. Solld bars, 1986; hatched bars, 1988.

sponsored plans have the largest networks of providers. What is interesting, though, is how quickly they have grown and how large they are. The number of hospitals in both types approximately tripled, and physicians more than doubled. Currently, the typical BC/BS plan contracts with 72 hospitals and 4700 physicians. The corresponding figures for the typical commercial insurer is 200 hospitals and 13000 physicians. This again dramatizes how national in scope PPOs have become, a far cry from the early 'mom and pop' operations.

\section{Cost containment mechanisms}

As discussed throughout the paper, we believe that the success of PPOs rests largely in their ability to control health care costs. Although our survey was not able to assess directly whether PPOs are saving money, we can gauge the likelihood that they are obtaining such savings by examining some of their characteristics.

There are four ways through which PPOs may elicit health care cost savings: (1) utilization review; (2) obtaining discounts from providers; (3) channeling patients to cost-effective providers; and (4) through incentives built into provider reimbursement mechanisms.

We believe that use of two of these cost containment mechanisms - channeling patients to efficient providers and employing provider reimbursement incentives are what can distinguish innovative PPOs from the rest. Peter Boland has stated that, 'The basic concept of PPOs is that consumers get better value for their health care dollar if they utilize more efficient doctors and hospitals', and further notes that, 'Physician practice patterns, rather than price, are the key to determining the consumption of medical services and the cost of health care' [24].

Of the four cost containment methods, discounts offer the least hope of controlling health care costs. Although there is nothing wrong with discounts, there is 
always a question as to whether true discounts are being elicited, or rather whether they are more illusory than real. More importantly, unlike the other three techniques, discounts do not conserve medical care resources. Rather, they are a redistribution in the burden of medical care costs from one party or payer to another.

Many would argue that employing strong utilization review is one of the most important steps that health plans can take to control costs. We would agree with this. However, even though utilization review is very important now in the United States, it can be carried on without PPOs. In fact, in a previous study of employers we found that 'managed' fee-for-service medicine, in which prospective utilization review techniques are employed, is one of the fastest growing sectors of the health care economy [25]. If a PPO relies solely on utilization review to control costs, it is unclear how it can be distinguished from managed fee-for-service care like that employed in most of the health care sector.

Channeling to cost-effective providers and reimbursement incentives, on the other hand, can distinguish a PPO from its PPO competitors (although it will make them look more like individual practice associations). If a network of providers truly does practice more cost-effective medicine, fewer resources will be expended on PPO patients. Even without such channeling, however, a reimbursement system can make providers act in a more cost-conscious manner, by rewarding them for the conservation of medical resources. DRGs provide such incentives in the inpatient setting by encouraging shorter lengths-of-stay and fewer ancillary services; capitation provides similar resource-conservation incentives in the physician sector [26].

We asked each respondent to rank these four methods in terms of their importance with regard to controlling health care costs. Table 2 shows the percentage of time each of the four methods was listed as the primary cost containment mechanism by the sampled PPOs. Admittedly, asking PPOs how they are saving money is not necessarily the best source of information on cost containment, and furthermore, we were unable to assess how much effort they are putting into each (e.g., how strong their utilization review programs are). Nevertheless, PPO rankings of their cost containment efforts do at least provide us with an idea of where PPOs think they are best able to achieve savings.

Hospital, physician, and insurer-sponsored PPOs stated that utilization review was their primary source of savings, whereas Blue Cross/Blue Shield plans listed discounts. Investor-sponsored PPOs, on the other hand, rarely listed utilization review; rather, their savings were split fairly evenly between the three other

Table 2

Primary cost-containment method listed by PPOs, by sponsor (percentage)

\begin{tabular}{lllll}
\hline Sponsor & Utilization review & Discounts & $\begin{array}{l}\text { Cost-effective } \\
\text { providers }\end{array}$ & $\begin{array}{l}\text { Reimburse } \\
\text { mechanisms }\end{array}$ \\
\hline Hospital & 48 & 18 & 18 & 16 \\
Physician & 60 & 10 & 20 & 10 \\
BC/BS & 28 & 44 & 11 & 17 \\
Insurer & 54 & 18 & 18 & 11 \\
Investor & 7 & 33 & 27 & 33 \\
\hline
\end{tabular}




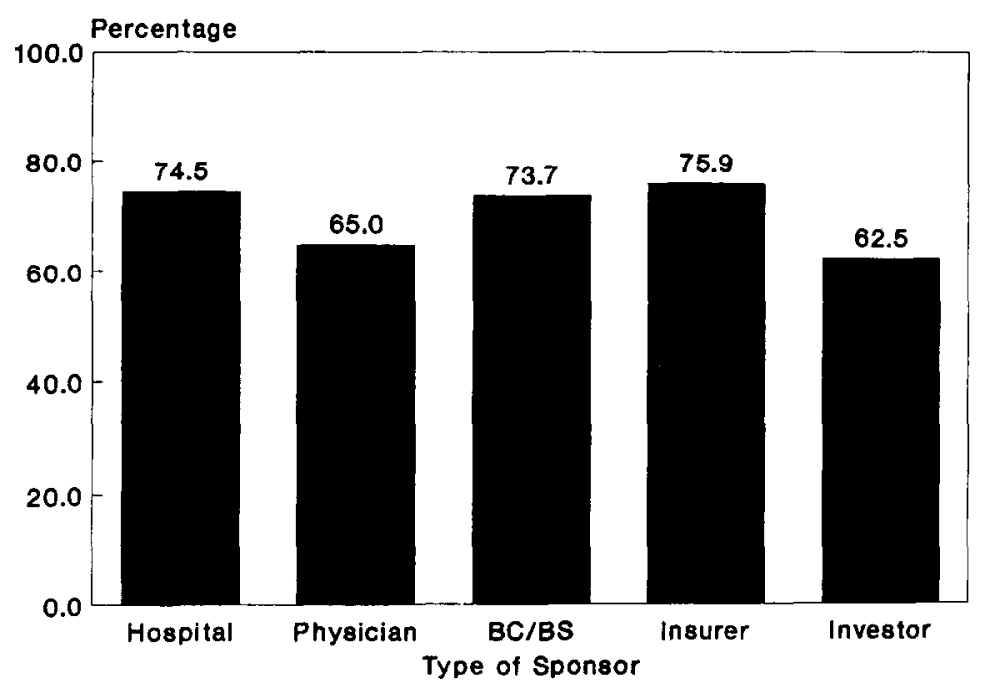

Fig. 6 Use of physician profiles, by sponsor.

mechanisms.

In our previous studies we had predicted that PPOs would increasingly depend on the more innovative cost-savings techniques, but this does not appear to be the case. The only sponsorship category that shows such a pattern are investorowned PPOs. Fully $60 \%$ reported one of these 'innovative' techniques as their primary cost-savings mechanisms, compared to less than $35 \%$ for each of the other sponsorship categories. One should not necessarily put too much stock into selfreported figures on savings such as these. For example, as shown below, investorsponsored plans were unlikely to use utilization data in selecting cost-effective providers. Nevertheless, the interest of investor-sponsored PPOs in innovative ways of containing health care costs may help explain their ability to continue to grow even without the advantages enjoyed by plans sponsored by the Blues and insurers.

In the following subsections, we examine each of the cost-savings techniques in more detail.

Utilization review. In previous studies we have reported that even as far back as 1985, utilization review techniques such as pre-admission certification of hospital stays, as well as concurrent and retrospective review of these stays, were already commonplace in PPO. In our 1988 survey we also asked PPOs whether they had developed profiles of physician services (see Fig. 6). If a PPO has a profile of each member physician's service provision activities, it has the ability to compare that physician with others and use that information to suggest changes in practice style. Unfortunately, the survey results provide no indication of the extent to which PPOs are using these profiles to elicit changes in physician behavior.

We were surprised to find that most PPOs in all sponsorship categories report that they were 'compiling and/or evaluating' physician profiles. In fact, there was very little dispersion between different types of plans: between $62 \%$ and $75 \%$ were 


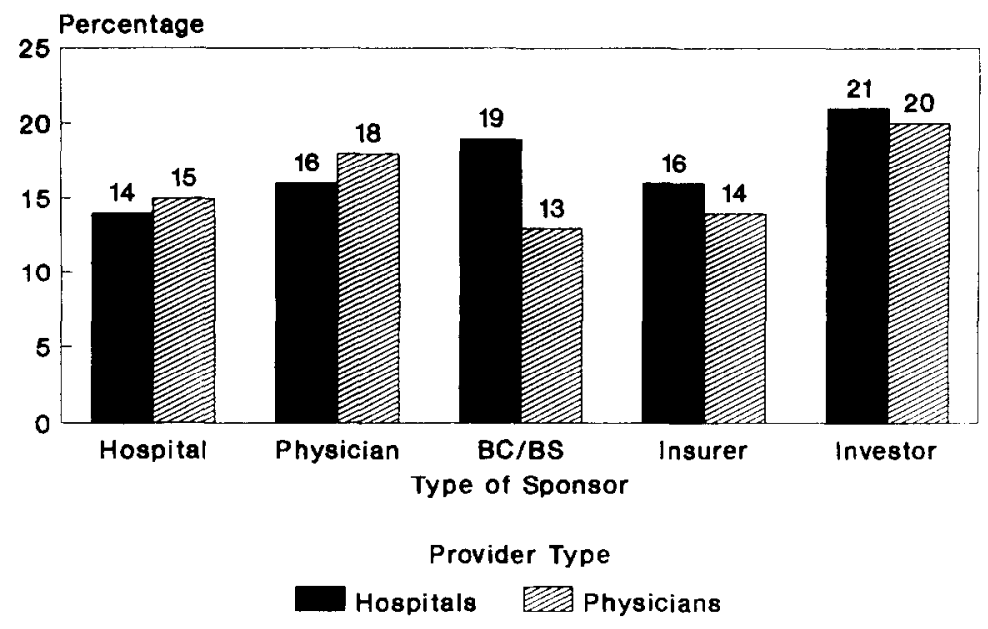

Flg. 7 Median hospltal and physician discounts reported by PPOs, by sponsor. Solid bars, hospitals; hatched bars, physicians.

doing this activity. This compares to less than half that were doing so in 1986 , although we reported at the time that 'the most dramatic development in utilization review involves physician profiling' [27]. However, comments from respondents indicate that this procedure may still be in its infancy. For example, a PPO may give providers information about outliers, but not be comfortable enough with the procedure to put much pressure on member physicians.

In summary, PPOs are continuing to rely heavily on utilization review, and are incorporating more and more techniques as they evolve. Perhaps the greatest issue for the future is not whether more techniques are employed, but how the information is used. If PPOs use physician profiles for purposes of trying to alter member providers' practice patterns, significant savings could result. However, we have doubts as to whether this will occur very much in the near future, since most PPOs have tried to be careful not to antagonize their provider panels.

Discounts. We asked PPOs the average discount they estimate they receive from preferred hospitals and physicians, in comparison to these providers' usual charges. Overall, the median reported discounts were about $17 \%$ from hospitals and $15 \%$ from physicians. Fig. 7 breaks this down by sponsorship category. The only pattern that emerges is that investor-sponsored plans report receiving somewhat higher discounts than do others.

Selection of Cost-Effective Providers. As a way of assessing PPOs' ability to select cost-effective providers, we asked respondents to list the data sources that they used in selecting both preferred hospitals and physicians. We then categorized their responses into whether these data allowed them to look at actual utilization figures for the provider. This would be possible, for example, if they listed 'claims data' or similar disaggregated utilization information. Fig. 8 shows these results.

Not surprisingly, Blue Cross/Blue Shield plans and commercial insurer PPOs 
were most likely to employ utilization data in provider selection. Over $80 \%$ of the Blues and almost $60 \%$ of the insurers said that they used utilization data for hospital selection. The same pattern emerged for physician selection, but utilization data were employed less often by all PPOs. Just over half of the Blue Cross/Blue Shield plans used such data, but only about a quarter of the insurers did so.

The other three sponsorship types did not tend to use such data in provider selection. This is probably due to the lack of availability of sufficient data. Earlier, we noted that investor-sponsored plans were most likely to claim that they achieved savings largely through selecting cost-effective providers. However, less than $40 \%$ used utilization data when choosing hospitals, and only about $12 \%$ used it for physician selection. Thus, they appear to have relied on their perceptions of costeffectiveness and perhaps their knowledge of the local market. One investorsponsored plan, for example, reported that it never contracted with university hospitals because it believed that they were 'rip-offs'.

Fig. 8 also indicates the relative disadvantage of physician-sponsored PPOs. Very few used utilization data in selecting providers. Earlier, we saw that they were least likely to employ physician profiling techniques (Fig. 6). These things may in part explain the fact that physician-sponsored PPOs are becoming less important in the market over time, as illustrated earlier in Fig. 2.

Provider reimbursement techniques. Fig. 9 shows the primary method of hospital reimbursement employed during our first survey, in 1985, and during 1988. The main pattern is that there has been a decline in the use of DRGs over the three year period. Whereas $15 \%$ of PPOs claimed this to be their dominant method in 1985 , only $7 \%$ said so in 1988 .

Fig. 10, which provides the findings on physician reimbursement, is very similar. In both years fee-schedules dominated. However, the primary technique that pro-

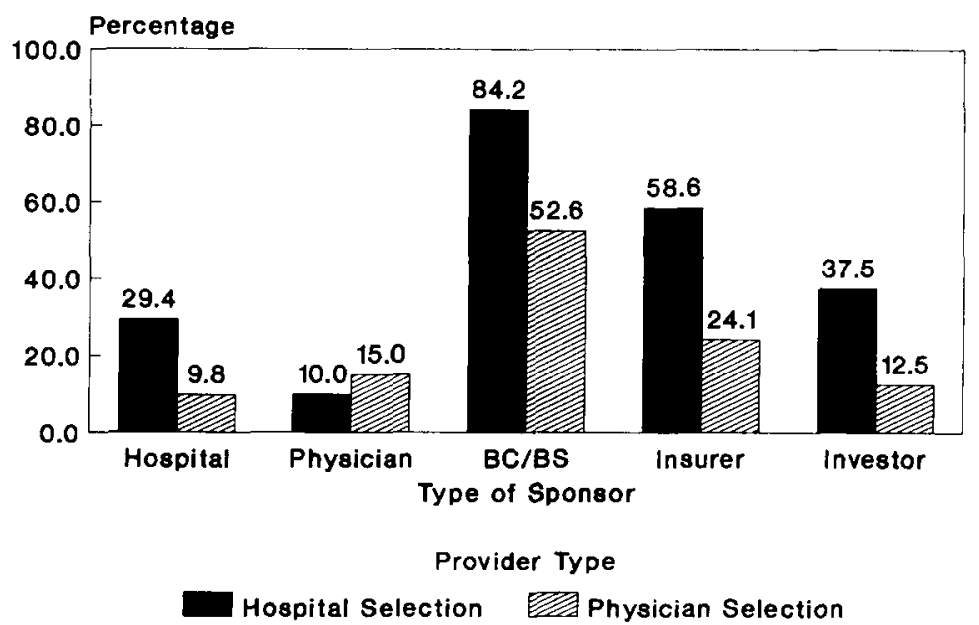

FIg. 8 Use of utilization or claims data for selecting preferred providers, by sponsor. Solld bars, hospital selection; hatched bars, physician selection. 


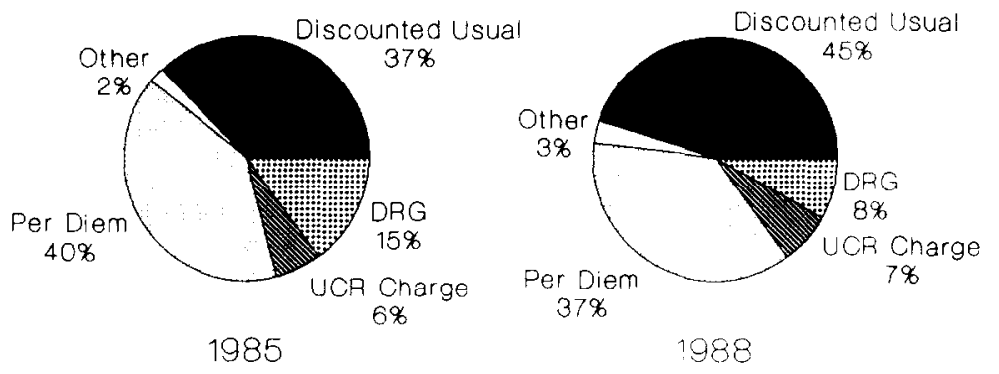

Flg. 9 Primary hospital reimbursement method during 1985 and 1988.

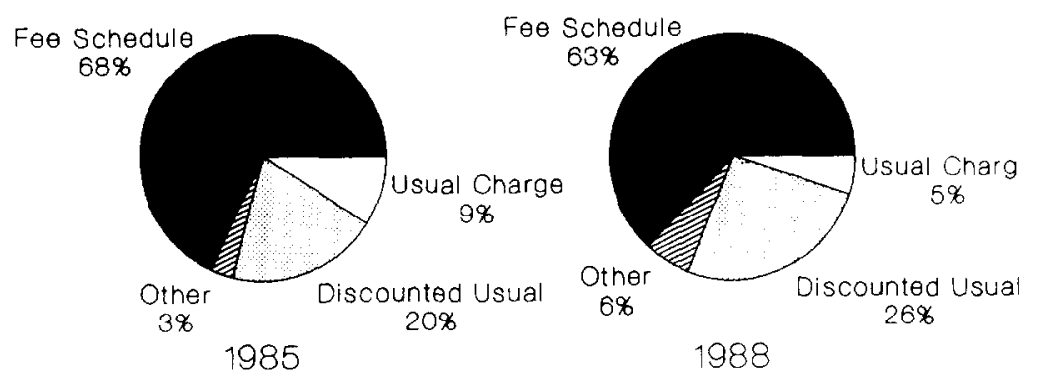

Fig- 10 Primary physician reimbursement method in 1985 and 1988.

vides an incentive to conserve resources is capitation - listed as part of the 'other' category. Although 'other' grew from $3 \%$ to $6 \%$ over the threc years, this was not due to any growth in capitation payments. During 1988 , only $1 \%$ of PPOs said that they relied largely on capitation for paying physicians.

Although it is easy to criticize PPOs for not moving to more innovative reimbursement systems, there are probably good reasons that they have retained their old methods. By paying hospitals by DRGs and physicians by capitation, PPOs might fear that they would antagonize their provider network, which is one of their main selling points. Furthermore, it is possible to build cost containment into the reimbursement system without resorting to capitation. One investor-sponsored plan indicated that it had developed its own fee-schedule that took all profits out of providing ancillary services. Another paid physicians on a DRG basis for their inpatient services.

\section{Conclusion}

In the last few years we have seen a resurgence in health care cost inflation. Controlling costs means reducing the rate of increase of resources that are going into the medical care sector. We must ask whether PPOs are succeeding in controlling medical resource inputs.

Most PPOs rely on utilization review and discounts to control health care costs. By themselves, discounts do little to reduce societal costs; that is, the amount of 
resources devoted to medical care. Utilization review is more promising, and the most important recent development in this regard is physician profiling. Most PPOs report that they are conducting this activity, but there is little evidence as yet that they are using the resulting information to affect the way in which medical care is delivered by providers. Furthermore, if utilization review is the major avenue by which PPOs save resources, one wonders why a PPO is needed to perform this activity, given the recent growth of 'managed' fee-for-service medicine outside of the PPO domain.

The two other forms of cost savings - selecting efficient providers and employing incentive reimbursement techniques - could, in our opinion, offer real promise in controlling costs. Our survey suggests that PPOs are not relying on reimbursement incentives. For example, the use of DRGs as a method of paying hospitals appears to have declined in the last few years. Although there may be other incentives available to hospitals to contain costs, DRGs appear to be the most successful of these to be adopted in recent years. We obtained mixed messages with regard to selection of cost-effective providers. Investor-sponsored PPOs showed the most interest in this area, but most such plans do not appear to have the necessary data to succeed. Blue Cross/Blue Shield plans and insurers, on the other hand, do have the requisite data, but they used these techniques less frequently.

One of the reasons that PPOs have grown so rapidly is that they are relatively inexpensive to develop and implement. Their recent growth certainly indicates that they will play a role in our health care environment for years to come. However, now that PPOs are an established part of our health care delivery system, they must show that they offer advantages over and above conventional health insurance plans, which themselves are moving towards more of a 'managed' care product by incorporating a number of utilization review requirements. PPOs must demonstrate the ability to control rising health care costs. To accomplish this, they will need to put more pressure on providers to use resources more sparingly. Otherwise, they may lose their market share to other forms of managed care.

\section{Acknowledgements}

The study was funded by the Health Insurance Association of America. All opinions and conclusions, however, are those of the authors and do not necessarily represent the views of the funding organization. We would like to thank an anonymous reviewer for helpful comments on an earlier draft of the article.

\section{References}

1 The distinction between HMOs and PPOs may be beginning to disappear. Feldman, R., Kralewski, J. and Dowd, B. [Health maintenance organizations: the beginning or the end? Health Services Research 24 (1989) 191-211] report that in the Minneapolis-St.Paul area, individual practice associations (IPAs) are beginning to look more like fee-for-service plans by providing coverage even when members go to out-of-plan providers, and that fee-for-service plans are incorporating 
more characteristics of HMOs such as providing expanded plan benefits. There are now even openended HMOs that use incentives similar to PPOs - lower out-of-pocket costs for users of in-plan providers. However, only about $2 \%$ of the 33 million HMO subscribers in 1989 were enrolled in these open ended plans [Friedman, G. Open-ended plans employ carrot, stick. Health Week, 3 (1989).]

2 De Lissovoy, G., Rice, T., Gabel, J. and Gelzer, G. Preferred provider organizations: one year later. Inquiry, 24 (1987) 127-135.

3 Hellinger, F. Selection bias in health maintenance organizations: analysis of recent evidence. Health Care Financing Review, 9 (1987) 55-63.

4 Federal Register 54:5142-5152, February 1, 1989).

5 Hester, J., Wouters, A. and Wright, N. Evaluation of a preferred provider organization. Milbank Quarterly. 65 (1987) 575-613.

6 Hosek, S., Marquis, M., Garnick, D. and Luft, H. The preferred provider organization study: summary of findings. The Rand Corporation. Santa Monica, CA (1989).

7 Ibid.

8 Rice, T., de Lissovoy, G., Gabel, J. and Ermann, D. The state of PPOs: results from a national survey. Health Affairs, 4 (1985) 25-40.

9 Gabel, J., Ermann, D., Rice, T. and de Lissovoy, G. The emergence and future of PPOs. Journal of Health Politics, Policy and Law, 11 (1986) 305-321.

10 De Lissovoy, G., Rice, T., Ermann, D. and Gabel, J. Preferred provider organizations: today's models and tomorrow's prospects. Inquiry, 23 (1986) 7-15.

11 See Note 2.

12 See Note 10.

13 See note 9.

14 American Medical Care and Review Association. Directory of preferred provider organizations and the industry report on PPO development. Bethesda, MD: AMCRA, (June 1988).

15 For example, out of a total population of 354 eligible non-commercial insurer-sponsored plans, 155 (44\%) were hospital-sponsored. Consequently, $44 \%$ of our total sample of non-insurer plans, or 53 $(0.44 \times 120)$, were hospital-sponsored plans. Of these 53,11 were determined to have eligibility figures greater than 100000 . The remaining 42 were drawn randomly from the remaining universe of 144 (155-11) eligible plans with fewer than 100000 individuals eligible to use PPO benefits. In total, 44\% of non-insurer interviews were hospital-sponsored PPOs, $17 \%$ were physician-sponsored, $16 \%$ each Blue Cross/Blue Shield and insurer-sponsoned, and $8 \%$ were 'other'.

16 Fifty-nine of these PPOs had more than 100000 eligibles. Approximately $30 \%$ of smaller PPOs were sampled as part of the survey.

17 See note 14.

18 Our formula for calculating the number of PPO eligibles (which was done separately by each of the sponsorship types) was to add eligibility figures in the PPOs with over 100000 members (which we sample at $100 \%$ ) to our estimate of total eligibility in smaller PPOs. The latter figure was derived by multiplying the sum of aggregate eligibility in the sampled PPOs by the inverse of their sampling rate.

19 See Note 5.

20 Gabel, J., DiCarlo, S., Fink, S. and De Lissovoy, G. Employer-sponsored health insurance in America: preliminary results from the 1988 survey. Washington, DC: Health Insurance Association of America (1989).

21 Gabel, J. et al. Trends in managed health care. Washington, D.C.: Health Insurance Association of America (February 1989).

22 Gabel, J., Jajich-Toth, C., De Lissovoy, G., Rice, T. and Cohen, H. The changing world of group health insurance. Health Affairs, 7 (1988) $48-65$.

23 See Note 14.

24 Boland, P. The illusion of discounts in the health care market. Health Affairs, 4 (1985) 93-97.

25 See note 21 .

26 Although there is not yet any empirical evidence on whether PPOs can control health care costs through selecting efficient providers and paying them in a way that encourages them to practice medicine more efficiently, the literature provides evidence that the reimbursement incentives appear to work. Early evidence from the Medicare DRG system indicates that the DRG system resulted in hospitals providing care more efficiently [Gutterman, S. et al. The first three years of Medicare 
prospective payment: an overview. Health Care Financing Review, 9 (1988) 67-77]. Furthermore, although the evidence is less clear-cut due to problems of self-selection bias, it appears that HMOs, which are paid on a capitation basis, also provide care more efficiently. However, the one controlled trial of the effect of HMOs on costs was conducted with data from only one prepaid group practice HMO [Manning. W.G. et al. A controlling trial of the effect of a prepaid group practice on use of services. New England Journal of Medicine, 310 (1984) 1505-1510]. The extent to which these results apply to IPAs and to other prepaid group practice HMOs has yet to be determined.

27 See Note 2. 